\title{
A new cubic nonconforming finite element on rectangles*
}

\author{
Zhaoliang Meng ${ }^{\dagger a}$, Zhongxuan Luo ${ }^{\mathrm{a}, \mathrm{b}}$, and Dongwoo Sheen ${ }^{\mathrm{c}}$ \\ ${ }^{a}$ School of Mathematical Sciences,Dalian University of Technology, Dalian, 116024, \\ China \\ ${ }^{\mathrm{b}}$ School of Software,Dalian University of Technology, Dalian, 116620, China \\ ${ }^{\mathrm{c}}$ Department of Mathematics 83 Interdisciplinary Program in Computational Sciences \\ \& Technology, Seoul National University, Seoul 151-747, Korea.
}

March 29, 2021

\begin{abstract}
A new nonconforming rectangle element with cubic convergence for the energy norm is introduced. The degrees of freedom (DOFs) are defined by the twelve values at the three Gauss points on each of the four edges. Due to the existence of one linear relation among the above DOFs, it turns out the DOFs are eleven. The nonconforming element consists of $P_{2} \oplus \operatorname{Span}\left\{x^{3} y-x y^{3}\right\}$. We count the corresponding dimension for Dirichlet and Neumann boundary value problems of second-order elliptic problems. We also present the optimal error estimates in both broken energy and $L_{2}(\Omega)$ norms. Finally, numerical examples match our theoretical results very well.
\end{abstract}

Keywords: nonconforming finite element; optimal error estimates; quadrilateral mesh

\section{Introduction}

It has been well known that the standard lowest order conforming elements can produce numerical locking and checker-board solutions in the approximation of solid and fluid mechanics problems: see for instance [3, 4, 6, 9, 15] and the references therein. An efficient approach to deal with this case is to employ the nonconforming element method, which has made a great impact on the development of finite element methods 1, 2, 5, 7, 8, 10 14, 16, 19, 20, 22, 23, 25, 27, 28.

To approximate PDEs using a nonconforming element of order $k$, one needs to impose the continuity of the moments up to order $k-1$ of the functions across all the interfaces of neighboring elements. This condition is known as the patch test [17]. In two dimensions, the patch test is equivalent to the continuity at the $k$ Gauss points located on each interface. This implies that a $P_{k}$-nonconforming element, if exists, must be continuous at the $k$ Gauss points on each edge. These points (completed with internal points for $k \geq 3$ ) can be used to define local Lagrange degrees of freedom (DOFs) on the simplex if $k$ is odd, but this construction is not possible if $k$ is even since there exists a lower-degree polynomial vanishing at all the Gauss points [14]. Thus suitable bubble functions are often employed to enrich the finite element space. Until now, the triangular nonconforming elements are well studied in the literature (see, [10, 14]), but the analysis of their quadrilateral counterparts is less complete.

Even though the triangular or tetrahedral meshes are popular to use, in some cases where the geometry of the problem has a quadrilateral nature, one wishes to use quadrilateral or hexahedral meshes with proper elements. For even $k$, the same trouble exists, that is, there also exists a lowerdegree polynomial vanishing at all the Gauss points. Again, some bubble functions are added to the

\footnotetext{
*This project is supported by NNSFC (Nos. 61033012,19201004,11271060,61272371), "the Fundamental Research Funds for the Central Universities", and NRF Nos. 2012-0000153.

${ }^{\dagger}$ Corresponding author: mzhl@ dlut.edu.cn
} 
finite element space [21]. Compared to the triangular case, another trouble for quadrilateral finite element is that the DOFs and corresponding polynomial space do not match. Usually the number of DOFs is bigger than the dimension of $P_{k}$. For example, for $k=1$ and $k=2$, the numbers of DOFs are 4 and 8, respectively, while the corresponding dimensions of $P_{k}$ are 3 and 6 , respectively. Therefore, some additional relations must be imposed or some special functions are added to the finite element space such that unisolvency can be satisfied, see [18, 21, 24].

The purpose of this paper is to develop a $P_{3}$-nonconforming element on rectangular meshes. We define the 12 Gauss points (3 Gauss points on every edge) as the DOFs. To obtain an optimal order error estimate, the finite element space must be carefully chosen such that any function in this space is a polynomial of degree no greater than 3 on every edge. Meanwhile, we also notice that the values on the 12 Gauss points satisfy a linear relation if the degree of a polynomial on every edge is no more 3 , which is a little different from the triangular mesh case. Thus we define our finite element space as $P_{3} \oplus \operatorname{Span}\left\{x^{3} y-x y^{3}\right\}$. Therefore, the number of DOFs is locally 11. We prove unisolvency and define three types of local and global bases, one of which is defined associated with vertices and the other two of which are defined associated with edges. Then we derive optimal error estimates for second-order elliptic problems in broken energy- and $L^{2}$-norms. Finally, numerical examples are provided, which match our theoretical result very well.

This paper is organized as follows. In Section 2 the $P_{3}$ nonconforming element is defined on rectangular meshes. The dimensions and basis functions for Dirichlet and Neumann problems are given. In section 3 interpolation operators are defined and optimal order error estimates are shown. In Section 4, numerical results for the elliptic problems are presented.

\section{The $P_{3}$ nonconforming element on rectangular mesh}

\subsection{The $P_{3}$-Nonconforming Quadrilateral Elements}

Take reference element as square $\widehat{R}=[-1,1]^{2}$. Denote by $V_{j}, j=1, \cdots, 4$, the vertices and by $g_{3 j-2}, g_{3 j-1}$ and $g_{3 j}$ the Gauss points on the edges $\overline{V_{j} V_{j+1}}, j=1, \cdots, 4$, with the identification $V_{1}=V_{5}$ (see Fig. 11):

$$
\begin{array}{rll}
g_{1}=(-\sqrt{3 / 5},-1), & g_{2}=(0,-1), & g_{3}=(\sqrt{3 / 5},-1), \\
g_{4}=(1,-\sqrt{3 / 5}), & g_{5}=(1,0), & g_{6}=(1, \sqrt{3 / 5}), \\
g_{7}=(\sqrt{3 / 5}, 1), & g_{8}=(0,1), & g_{9}=(-\sqrt{3 / 5}, 1), \\
g_{10}=(-1, \sqrt{3 / 5}), & g_{11}=(-1,0), & g_{12}=(-1, \sqrt{3 / 5}) .
\end{array}
$$

Denoting by $P_{k}(\widehat{R})$ the space of polynomials of degree $\leq k$ on $\widehat{R}$, set

$$
\widehat{\mathbb{P}}=P_{3}(\widehat{R}) \oplus \operatorname{Span}\left\{x^{3} y-x y^{3}\right\}, \quad \text { with } \operatorname{dim}(\widehat{\mathbb{P}})=11 .
$$

The space $\widehat{\mathbb{P}}$ will be our nonconforming finite element space on $\widehat{R}$ with appropriate degrees of freedom that will be defined soon. Before proceeding, we notice the following simple result.

Lemma 1. The following relationship holds:

$$
3 p(-1)+3 p(1)-5 p(\sqrt{3 / 5})-5 p(-\sqrt{3 / 5})+4 p(0)=0 \quad \forall p \in P_{3}(\mathbb{R}) .
$$

Proof. Let $p \in P_{3}(\mathbb{R})$. Then any fourth order difference quotient of $p(x)$ vanishes. The fourth order difference quotient of $p(x)$ at points $x_{1}=-1, x_{2}=-\sqrt{3 / 5}, x_{3}=0, x_{4}=\sqrt{3 / 5}$, and $x_{5}=1$ can be expressed by

$$
\sum_{i=1}^{5} \frac{p\left(x_{i}\right)}{\left(x_{i}-x_{1}\right) \cdots\left(x_{i}-x_{i-1}\right)\left(x_{i}-x_{i+1}\right) \cdots\left(x_{i}-x_{5}\right)} .
$$




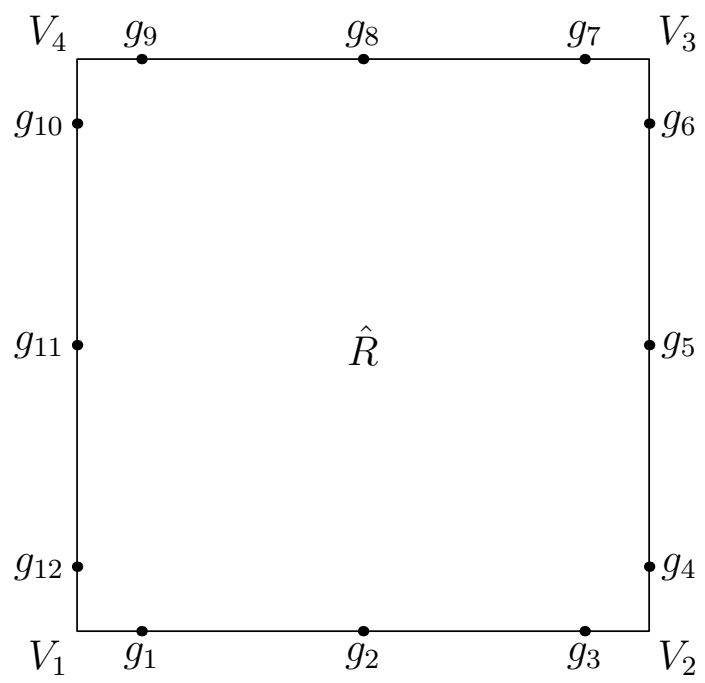

Figure 1: The reference rectangle $\widehat{R}$

A simple computation derives the desired result.

As an immediate consequence of Lemma 1, we have the following proposition.

Proposition 1. The following relationship holds:

$$
\begin{aligned}
& 4\left(\varphi\left(g_{2}\right)+\varphi\left(g_{8}\right)\right)-5\left(\varphi\left(g_{1}\right)+\varphi\left(g_{3}\right)+\varphi\left(g_{7}\right)+\varphi\left(g_{9}\right)\right) \\
& \quad=4\left(\varphi\left(g_{5}\right)+\varphi\left(g_{11}\right)\right)-5\left(\varphi\left(g_{4}\right)+\varphi\left(g_{6}\right)+\varphi\left(g_{10}\right)+\varphi\left(g_{12}\right)\right) \quad \forall \varphi \in \widehat{\mathbb{P}} .
\end{aligned}
$$

Proof. Notice that $\varphi$ is a polynomial of degree no greater than 3 on any edge of $\widehat{R}$. The result follows immediately from Lemma 1.

Lemma 2. Suppose that $\varphi \in \widehat{\mathbb{P}}$ vanishes at the twelve Gauss points $g_{j}, j=1, \cdots, 12$. Then $\varphi=0$ in $\widehat{R}$.

Proof. Let $p \in P_{3}(\mathbb{R})$ vanish at vanishes at the twelve Gauss points $g_{j}, j=1, \cdots, 12$. It is easy to check that $\varphi_{1}(x, y)=x^{2}+y^{2}-8 / 5$ vanishes at the eight Gauss points $g_{3 j-2}, g_{3 j}, j=1,2,3,4$. By a simple polynomial division, $\varphi(x, y)$ can be written as

$$
\varphi(x, y)=\varphi_{1}(x, y)\left(a_{0} x y+a_{1} x+a_{2} y+a_{3}\right)+x r_{1}(y)+r_{2}(y)
$$

where $r_{1}(y)$ and $r_{2}(y)$ take the following form:

$$
r_{1}(y)=-2 a_{0} y^{3}+b_{1} y^{2}+b_{2} y+b_{3} \quad \text { and } r_{2}(y)=c_{0} y^{3}+c_{1} y^{2}+c_{2} y+c_{3} .
$$

All the coefficients $a_{j}, b_{j}, c_{j}$ are yet to be determined. It follows from $\varphi( \pm \sqrt{3 / 5}, 1)=\varphi_{1}( \pm \sqrt{3 / 5}, 1)=0$ that

$$
\sqrt{3 / 5} r_{1}(1)+r_{2}(1)=0, \quad \text { and } \quad-\sqrt{3 / 5} r_{1}(1)+r_{2}(1)=0,
$$

which implies that $r_{1}(1)=r_{2}(1)=0$. Similarly, if follows from $\varphi( \pm \sqrt{3 / 5},-1)=\varphi_{1}( \pm \sqrt{3 / 5},-1)=0$ that $r_{1}(-1)=r_{2}(-1)=0$. Next, it follows from $\varphi( \pm 1, \sqrt{3 / 5})=\varphi_{1}( \pm 1, \sqrt{3 / 5})=0$ that $r_{1}( \pm \sqrt{3 / 5})=$ $r_{2}( \pm \sqrt{3 / 5})=0$. Since $r_{1}$ and $r_{2}$ are polynomials of degree no more 3 , which leads to $r_{1}(y)=r_{2}(y)=0$. Hence $\varphi(x, y)$ must have the following form

$$
\varphi(x, y)=\varphi_{1}(x, y)\left(a_{1} x+a_{2} y+a_{3}\right) .
$$

Then the four additional conditions $\varphi\left(g_{3 j-1}\right)=0, j=1,2,3,4$, lead to $\varphi(x, y)=0$, which completes the proof. 
Due to proposition 1 and Lemma 2, we have the unisolvency result.

Proposition 2. A function $\varphi \in \widehat{\mathbb{P}}$ is uniquely determined by $\varphi\left(g_{j}\right), j=1,2, \cdots, 12$, which satisfy the relation (2).

Denote by $M_{j}, j=1,2,3,4$, the four midpoints of four edges. Obviously, $M_{j}$ is one of three Gauss points on $j$ th edge and hence the other two Gauss points on $j$ th edge can also be denoted by $M_{j}^{+}$and $M_{j}^{-}$. For example, in Fig. 1 $M_{1}^{+}=g_{1}$ and $M_{1}^{-}=g_{3}$. For $1 \leq j \leq 4$, define $\varphi_{j}^{V}, \varphi_{j}^{E_{+}}$and $\varphi_{j}^{E_{-}} \in \widehat{\mathbb{P}}$ (see Fig. 2) by

$$
\varphi_{j}^{V}\left(g_{k}\right)= \begin{cases}1, & k=3 j-3,3 j-2, \\ 0, & \text { otherwise, }\end{cases}
$$

(with the identification $g_{0}=g_{12}$ ), and

$$
\varphi_{j}^{E_{+}}\left(g_{k}\right)= \begin{cases}4, & g_{k}=M_{j}^{+}, \\ 5, & g_{k}=M_{j}, \\ 0, & \text { otherwise }\end{cases}
$$

and

$$
\varphi_{j}^{E_{-}}\left(g_{k}\right)= \begin{cases}5, & g_{k}=M_{j}, \\ 4, & g_{k}=M_{j}^{-}, \\ 0, & \text { otherwise }\end{cases}
$$

We then have the following result.

Lemma 3. $\operatorname{Span}\left\{\varphi_{j}^{V}, \varphi_{j}^{E_{+}}, \varphi_{j}^{E_{-}}, j=1,2,3,4\right\}=\widehat{\mathbb{P}}$. Indeed, any eleven of $\varphi_{j}^{V}, \varphi_{j}^{E_{+}}, \varphi_{j}^{E_{-}}, j=1,2,3,4$, $\operatorname{span} \widehat{\mathbb{P}}$.

The proof of this lemma is similar to that of Lemma 2.3 in [21], which will be omitted.

We are now in a position to state the definition of $P_{3}$-nonconforming element on a rectangle as follows.

Definition 1. The $P_{3}$-nonconforming element on rectangle is defined by $\left(\widehat{R}, \widehat{\mathbb{P}}_{\widehat{R}}, \widehat{\sum}_{\widehat{R}}\right)$, where

- $\widehat{R}$ is a rectangle,

- $\widehat{\mathbb{P}}_{\widehat{R}}=\widehat{\mathbb{P}}$ is the finite element space, and

- $\widehat{\sum}_{\widehat{R}}=\left\{\varphi\left(g_{j}\right), j=1,2, \cdots, 12\right.$, such that Eq. (2) holds for all $\left.\varphi \in \widehat{\mathbb{P}}_{\widehat{R}}\right\}$ is the degrees of freedom.

The following patch test lemma is immediate since any $p \in \widehat{\mathbb{P}}$ is of degree $\leq 3$ on an edge.

Lemma 4. Let $E_{j}$ denote the face containing the midpoint $M_{j}$ and the other two Gauss points $M_{j}^{+}$ and $M_{j}^{-}$. If $p \in \widehat{\mathbb{P}}, p\left(M_{j}\right)=p\left(M_{j}^{+}\right)=p\left(M_{j}^{-}\right)=0$, then

$$
\int_{E_{j}} p(x, y) q(x, y) \mathrm{d} \sigma=0
$$

for all $q \in Q_{2}\left(E_{j}\right)$.

Remark 1. For actual computation, the local finite element can be alternatively given by $\left\{\sum_{j=1}^{4}\left(c_{j}^{V} \varphi_{j}^{V}+\right.\right.$ $\left.\left.c_{j}^{E_{+}} \varphi_{j}^{E_{+}}+c_{j}^{E_{-}} \varphi_{j}^{E_{-}}\right)\right\}$.

Remark 2. In theory, if $x^{3} y-x y^{3}$ is replaced by $x^{3} y, x y^{3}$ or $x^{3} y+x y^{3}$, the unisolvency like Proposition 2 holds. But the former two choices are lack in symmetry. As for the third choice $x^{3} y+x y^{3}$, it turns out to be numerically during the computation of the bases since the corresponding coefficient matrices are nearly ill-conditioned (the determinants are near to zero). 


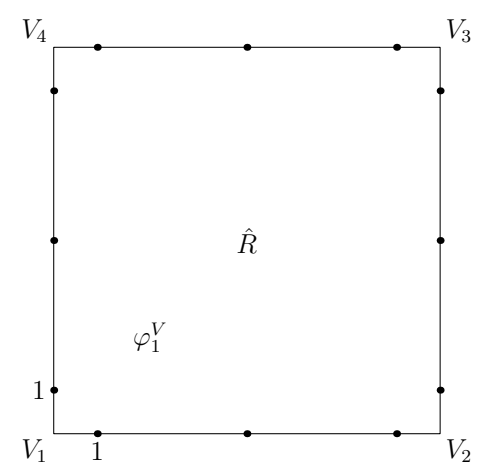

(a)

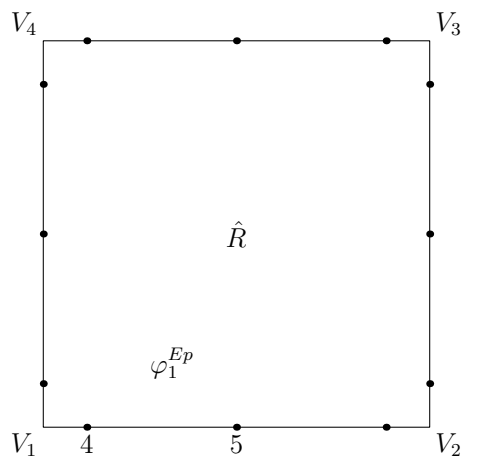

(b)

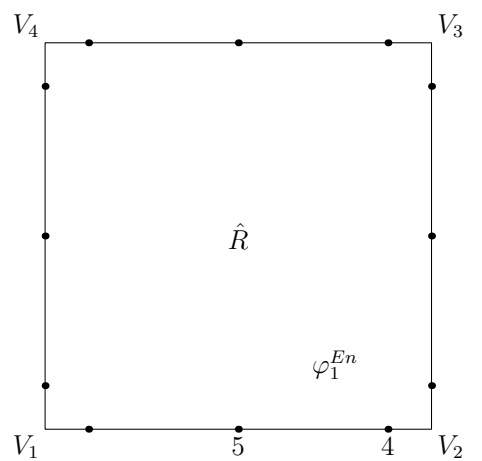

(c)

Figure 2: Three types of basis function. (a) The vertex-based basis function associated with vertex $V_{1}$. (b) The fist type of the edge-based function associated with edge $E_{1}$. (c) The second type of the edge-based function associated with edge $E_{1}$. 
Let us proceed to define our $P_{3}$-nonconforming element space. Assume that $\Omega \in \mathbb{R}^{2}$ is a parallelogram domain with boundary $\Gamma$. Let $\left(\mathscr{T}_{h}\right)_{h>0}$ be a regular family of triangulation of $\Omega$ into parallelograms $R_{j}, j=1,2, \cdots, N_{R}$, where $h=\max _{R \in \mathscr{T}_{h}} h_{R}$ with $h_{R}=\operatorname{diam}(R)$. For each $R \in \mathscr{T}_{h}$, let $F_{R}: \widehat{R} \rightarrow \mathbb{R}^{2}$ be an invertible affine mapping such that

$$
R=F_{R}(\widehat{R}),
$$

and denote $\widehat{\phi}_{R}=\widehat{\phi} \circ F_{R}^{-1}: R \rightarrow \mathbb{R}$ for all $\widehat{\phi} \in \widehat{\mathbb{P}}$, whose collection will be designated by

$$
\mathbb{P}_{R}=\operatorname{Span}\left\{\phi_{R}, \widehat{\phi} \in \widehat{\mathbb{P}}\right\} .
$$

For a given triangulation $\mathscr{T}_{h}$ of $\Omega$, let $N_{V}, N_{E}, N_{R}$, and $N_{G}$ denote the numbers of vertices, edges, rectangles, and Gauss points, respectively. Then set

$$
\begin{aligned}
& \mathscr{V}_{h}=\left\{V_{1}, V_{2}, \cdots, V_{N_{V}}\right\}: \text { the set of all vertices of } R \in \mathscr{T}_{h}, \\
& \mathscr{E}_{h}=\left\{E_{1}, E_{2}, \cdots, E_{N_{E}}\right\}: \text { the set of all edges of } R \in \mathscr{T}_{h}, \\
& \mathscr{G}_{h}=\left\{g_{1}, g_{2}, \cdots, g_{N_{G}}\right\}: \text { the set of all Gauss points on } \mathscr{E}_{h} \in \mathscr{T}_{h} \\
& \mathscr{M}_{h}=\left\{M_{i} \in E_{i}, i=1,2, \cdots, N_{E}\right\}: \text { the set of all Midpoints on } \mathscr{E}_{h} \in \mathscr{T}_{h} .
\end{aligned}
$$

In particular, let $N_{V}^{i}, N_{E}^{i}$, and $N_{G}^{i}$ denote the numbers of interior vertices, edges, and Gauss points of $R \in \mathscr{T}_{h}$, respectively.

For a function $f$ defined in $\Omega$, denote by $f_{j}$ its restriction to $R_{j}$, and $E_{j k}$ the interface between $R_{j}$ and $R_{k}$. Similarly, $g_{j k}, k=1,2,3$, will mean the Gauss points on $\Gamma_{j}=\partial R_{j} \cap \partial \Omega$ and $g_{j k l}, l=1,2,3$, will be the Gauss points on $E_{j k}$. We are now in a position to define the following nonconforming finite element spaces.

$$
\begin{aligned}
& \mathscr{N} \mathscr{C}^{h}=\left\{\varphi: \Omega \rightarrow \mathbb{R}|\varphi|_{R} \in \mathbb{P}_{R}, \forall R \in \mathscr{T}_{h}, \varphi \text { is continuous at the Gauss points } g \in \mathscr{G}_{h}\right\}, \\
& \mathscr{N} \mathscr{C}_{0}^{h}=\left\{\varphi \in \mathscr{N} \mathscr{C}^{h} \mid \varphi(g)=0, \text { for all Gauss points } g \in \mathscr{G}_{h} \cap \Gamma\right\} .
\end{aligned}
$$

For each vertex $V_{j} \in \mathscr{V}_{h}$, denote by $\mathscr{E}_{h}(j)$ and $\mathscr{G}_{h}(j)$ the set of all edges $E \in \mathscr{E}_{h}$ with one of the endpoints being $V_{j}$ and the set of Gauss points nearest to $V_{j}$ among the three Gauss points on $E$ for all $E \in \mathscr{E}_{h}(j)$. For $M_{j} \in E_{j}$, if $g_{i}$ and $g_{k}$ are two other Gauss points and $i<k$, we also denote these two Gauss points by $M_{j}^{+}$and $M_{j}^{-}$, respectively. We then define the three types of functions in $\mathscr{N}_{\mathscr{C}^{h}}$, which serve as global bases for the nonconforming finite element spaces.

Definition 2. The first type of functions are associated with vertices. Define $\varphi_{j}^{V} \in \mathscr{N}^{h}, j=$ $1,2, \cdots, N_{V}$, by

$$
\varphi_{j}^{V}= \begin{cases}1, & g_{k} \in \mathscr{G}_{h}(j) \\ 0, & \text { otherwise }\end{cases}
$$

Next define the second type of functions associated with edges $E_{j} \in \mathscr{E}:$ define $\varphi_{j}^{E_{+}} \in \mathscr{N} \mathscr{C}^{h}, j=$ $1,2, \cdots, N_{E}$, by

$$
\varphi_{j}^{E_{+}}= \begin{cases}5, & g_{k}=M_{j} \\ 4, & g_{k}=M_{j}^{+} \\ 0, & \text { otherwise }\end{cases}
$$

The last type of functions are also associated with edges $E_{j} \in \mathscr{E}:$ define $\varphi_{j}^{E_{-}} \in \mathscr{N}^{h}, j=1,2, \cdots, N_{E}$, by

$$
\varphi_{j}^{E_{-}}= \begin{cases}5, & g_{k}=M_{j} \\ 4, & g_{k}=M_{j}^{-} \\ 0, & \text { otherwise }\end{cases}
$$


Similarly, define the three types of functions which will serve as global basis functions for $\mathscr{N}_{0}^{h}$ with those for $\mathscr{N}_{\mathscr{C}^{h}}$ excluding $\varphi_{j}^{V}$ 's which are associated with boundary vertices and $\varphi_{j}^{E_{+}}, \varphi_{j}^{E_{-}}$'s which are associated with boundary edges.

Now let us present the dimensions for the nonconforming finite element spaces.

Theorem 1. $\operatorname{dim}\left(\mathscr{N} \mathscr{C}^{h}\right)=N_{V}+2 N_{E}-1$. Let $\varphi_{j}^{V}, j=1,2, \cdots, N_{V}$ and $\varphi_{j}^{E_{+}}, \varphi_{j}^{E_{-}}, j=1,2, \cdots, N_{E}$

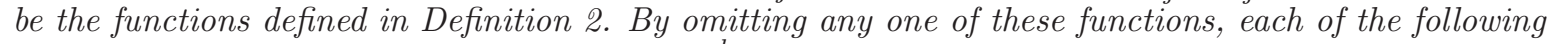
three sets forms global basis functions for $\mathscr{N}_{C^{h}}$ :

$$
\begin{aligned}
& \mathscr{B}_{1}=\left\{\varphi_{1}^{V}, \varphi_{2}^{V}, \cdots, \varphi_{N_{V}-1}^{V}, \varphi_{j}^{E_{+}}, \varphi_{j}^{E_{-}}, j=1,2, \cdots, N_{E}\right\}, \\
& \mathscr{B}_{2}=\left\{\varphi_{j}^{V}, j=1,2, \cdots, N_{V}, \varphi_{1}^{E_{+}}, \varphi_{2}^{E_{+}}, \cdots, \varphi_{N_{E}-1}^{E_{+}}, \varphi_{i}^{E_{-}}, i=1,2, \cdots, N_{E}\right\},
\end{aligned}
$$

and

$$
\mathscr{B}_{3}=\left\{\varphi_{j}^{V}, j=1,2, \cdots, N_{V}, \varphi_{i}^{E_{+}}, i=1,2, \cdots N_{E}, \varphi_{1}^{E_{-}}, \varphi_{2}^{E_{-}}, \cdots, \varphi_{N_{E}-1}^{E_{-}}\right\}
$$

Theorem 2. $\operatorname{dim}\left(\mathscr{N} \mathscr{C}_{0}^{h}\right)=N_{V}^{i}+2 N_{E}^{i} \cdot \mathscr{B}=\left\{\varphi_{i}^{V}, i=1,2, \cdots, N_{V}^{i}, \varphi_{j}^{E_{+}}, \varphi_{j}^{E_{-}}, j=1,2, \cdots, N_{E}^{i}\right\}$ forms a set of global basis functions for $\mathscr{N}_{C_{0}^{h}}^{h}$.

The proofs of the above theorems are quite similar to those in the literature [21], and thus omitted. Here we remark that our finite element space is a little different from those in the literature [18, 21]. Those finite element spaces are nothing but conforming element spaces enriched by some suitable bubble function spaces. Thus the idea of Fortin and Soulie [14] is not applicable here.

\section{The interpolation operator and convergence analysis}

In this section we will define an interpolation operator and analyze convergence in the case of Dirichlet problem. The case of Neumann problem is quite similar and the results will be briefly stated with their details being omitted.

Denote by $(\cdot, \cdot)$ the $L^{2}(\Omega)$ inner product and $(f, v)$ will be understood as the duality pairing between $H^{-1}(\Omega)$ and $H_{0}^{1}(\Omega)$, which is an extension of the duality paring between $L^{2}(\Omega)$. By $\|\cdot\|_{k}$ and $|\cdot|_{k}$ we adopt the standard notations for the norm and seminorm for the Sobolev spaces $H^{k}(\Omega)$. Consider then the following Dirichlet problem:

$$
\begin{aligned}
-\nabla \cdot(\boldsymbol{\alpha} \nabla u)+\beta u & =f, \quad \Omega, \\
u & =0, \quad \Gamma,
\end{aligned}
$$

with $\boldsymbol{\alpha}=\left(\alpha_{j k}\right), \alpha_{j k}, \beta \in L^{\infty}(\Omega), j, k=1,2,0<\alpha_{*}|\boldsymbol{\xi}|^{2} \leq \boldsymbol{\xi}^{t} \boldsymbol{\alpha}(x) \boldsymbol{\xi} \leq \alpha^{*}|\boldsymbol{\xi}|^{2}<\infty, \boldsymbol{\xi} \in \mathbb{R}^{2}, \beta(x) \geq 0, x \in$ $\Omega$, and $f \in H^{1}(\Omega)$. We will assume that the coefficients are sufficiently smooth and that the elliptic problem (7) has an $H^{4}(\Omega)$-regular solution. The weak problem is then given as usual: find $u \in H_{0}^{1}(\Omega)$ such that

$$
a(u, v)=(f, v), \quad v \in H_{0}^{1}(\Omega),
$$

where $a: H_{0}^{1}(\Omega) \times H_{0}^{1}(\Omega) \rightarrow \mathbb{R}$ is the bilinear form defined by $a(u, v)=(\boldsymbol{\alpha} \nabla u, \nabla v)+(\beta u, v)$ for all $u, v \in H_{0}^{1}(\Omega)$. Our nonconforming method for Problem (7) states as follows: find $u_{h} \in \mathscr{N}_{0}^{h}$ such that

$$
a_{h}\left(u_{h}, v_{h}\right)=\left(f, v_{h}\right), \quad v_{h} \in \mathscr{N} \mathscr{C}_{0}^{h},
$$

where

$$
a_{h}(u, v)=\sum_{R \in \mathscr{T}_{h}} a_{R}(u, v),
$$

with $a_{R}$ being the restriction of $a$ to $R$. 
For a given rectangle $R \in \mathscr{T}_{h}$, define the local interpolation operator $\Pi_{R}: W^{1, p}(R) \cap H_{0}^{1}(\Omega) \longrightarrow$ $\mathbb{P}_{R}, p>1$, by

$$
\Pi_{R} \phi\left(g_{i}\right)=\phi\left(g_{i}\right),
$$

for all Gauss points on the edges of $R$. The global interpolation operator $\Pi_{h}: W^{1, p}(\Omega) \cap H_{0}^{1}(\Omega) \rightarrow \mathscr{N}_{0}^{h}$ is then defined through the local interpolation operator $\Pi_{R}$ by $\left.\Pi_{h}\right|_{R}=\Pi_{R}$ for all $R \in \mathscr{T}_{h}$. Since $\Pi_{h}$ preserves $P_{3}$ for all $R \in \mathscr{T}_{h}$, it follows from the Bramble-Hilbert Lemma that

$$
\begin{array}{r}
\sum_{R \in \mathscr{T}_{h}}\left\|\phi-\Pi_{h} \phi\right\|_{L^{2}(R)}+h \sum_{R \in \mathscr{T}_{h}}\left\|\phi-\Pi_{h} \phi\right\|_{H^{1}(R)} \leq C h^{k}|\phi|_{H^{k}(\Omega)}, \\
\phi \in W^{k, p}(\Omega) \cap H_{0}^{1}(\Omega), 2 \leq k \leq 4 .
\end{array}
$$

Denote $E_{j k}=\left(\partial R_{j} \cap \partial R_{k}\right)^{\circ}$ for all $R_{j}, R_{k} \in \mathscr{T}_{h}$ and by $\Gamma_{j}$ the boundary face $\left(\partial R_{j} \cap \partial \Omega\right)^{\circ}$ of $\mathscr{T}_{h}$. Then define

$$
\Lambda^{h}=\left\{\lambda\left|\lambda_{j k}=\lambda\right|_{E_{j k}} \in P_{2}\left(E_{j k}\right) ; \lambda_{j k}+\lambda_{k j}=0 ; \lambda_{j}=\left.\lambda\right|_{\Gamma_{j}} \in P_{2}\left(\Gamma_{j}\right)\right\},
$$

where $P_{2}(E)$ denotes the set of quadratic polynomials on the face $E$. Also define the projection $P_{h}: H^{3 / 2}(\Omega) \rightarrow \Lambda^{h}$ such that

$$
\left\langle\boldsymbol{\nu}_{j}^{T} \boldsymbol{\alpha} \nabla v_{j}-P_{h} v_{j}, z\right\rangle_{E}=0 \text { for all } z \in P_{2}(E) \text { for all } E \in \mathscr{E}_{h},
$$

where $v_{j}=\left.v\right|_{R_{j}}$ and $\boldsymbol{\nu}_{j}^{T}$ is the transpose of the unit outward normal to $R_{j}$. Then we have the following standard polynomial approximation result:

$$
\left\{\sum_{j}\left\|\boldsymbol{\nu}_{j}^{T} \boldsymbol{\alpha} \nabla v_{j}-P_{h} v_{j}\right\|_{L^{2}\left(\partial R_{j}\right)}^{2}\right\}^{1 / 2} \leq C h^{k-3 / 2}\|v\|_{k}, \quad \forall v \in W^{k, p}(\Omega), k=2,3,4, p>3 .
$$

Since $w_{j}-w_{k}$ has zero values at the Gauss points on $E_{j k}$ for all $w \in \mathscr{N}_{0}^{h}$ and the 3-point Gauss quadrature is exact on polynomials of degree no more than 5 , the following useful orthogonality holds. (See also Lemma 4)

Lemma 5. If $u \in H^{3 / 2}(\Omega)$, then the following equality holds:

$$
\left\langle P_{h} u_{j}, w_{j}\right\rangle_{E_{j k}}+\left\langle P_{h} u_{k}, w_{k}\right\rangle_{E_{k j}}=\left\langle P_{h} u_{j}, w_{j}-w_{k}\right\rangle_{E_{j k}}=0 \quad \text { for all } w \in \mathscr{N} \mathscr{C}_{0}^{h} .
$$

Denote the broken energy norm $\|\cdot\|_{h}$ on $\mathscr{N}_{\mathscr{C}^{h}}+H^{1}(\Omega)$ by

$$
\|\varphi\|_{h}=a_{h}(\varphi, \varphi)^{1 / 2} \quad \text { for all } \varphi \in \mathscr{N} \mathscr{C}^{h}+H^{1}(\Omega) .
$$

We now consider the energy-norm error estimate and first consider the following Strang lemma [26].

Lemma 6. Let $u \in H^{1}(\Omega)$ and $u_{h} \in \mathscr{N} \mathscr{C}_{0}^{h}$ be the solutions of Eq. (8) and Eq. (9), respectively. Then

$$
\left\|u-u_{h}\right\|_{h} \leq c\left\{\inf _{v \in \mathscr{N} \mathscr{C}_{0}^{h}}\|u-v\|_{h}+\sup _{w \in \mathscr{N} \mathscr{C}_{0}^{h}} \frac{\left|a_{h}(u, w)-\langle f, w\rangle\right|}{\|w\|_{h}}\right\} .
$$

Assume sufficient regularity such that $u \in H^{4}$. Due to (10), the first term in the right side of (12) is bounded by

$$
\inf _{v \in \mathscr{N}_{0}^{h}}\|u-v\|_{h} \leq\left\|u-\Pi_{h} u\right\|_{h} \leq c h^{s}|u|_{H^{s+1}(\Omega)}, 1<s \leq 3
$$

In order to bound the second term of the right side of (12) which denotes the consistency error, integrate by parts elementwise so that

$$
a_{h}(u, w)-\langle f, w\rangle=\sum_{j}\left\langle\boldsymbol{\nu}_{j}^{T} \boldsymbol{\alpha} \nabla u_{j}, w\right\rangle_{\partial R_{j} \backslash \Gamma_{j}}
$$




$$
\begin{aligned}
& =\sum_{j}\left\langle\boldsymbol{\nu}_{j}^{T} \boldsymbol{\alpha} \nabla u_{j}-P_{h} u_{j}, w\right\rangle_{\partial R_{j} \backslash \Gamma_{j}} \\
& =\sum_{j}\left\langle\boldsymbol{\nu}_{j}^{T} \boldsymbol{\alpha} \nabla u_{j}-P_{h} u_{j}, w-m_{j}\right\rangle_{\partial R_{j} \backslash \Gamma_{j}}
\end{aligned}
$$

where $m_{j} \in Q_{2}\left(R_{j}\right)$ is a biquadratic polynomial on $R_{j}$. In particular, if $m_{j}$ is chosen as the $Q_{2}$ projection of $w$ in $R_{j}$, due to the trace theorem, (10) and (11), we get

$$
\begin{aligned}
\left|\sum_{j}\left\langle\boldsymbol{\nu}_{j}^{T} \boldsymbol{\alpha} \nabla u_{j}-P_{h} u_{j}, w-m_{j}\right\rangle_{\partial R_{j}}\right| & \leq \sum_{j}\left\|\boldsymbol{\nu}_{j}^{T} \boldsymbol{\alpha} \nabla u_{j}-P_{h} u_{j}\right\|_{L_{2}\left(\partial R_{j}\right)}\left\|w-m_{j}\right\|_{L_{2}\left(\partial R_{j}\right)} \\
& \leq\left(\sum_{j}\left\|\boldsymbol{\nu}_{j}^{T} \boldsymbol{\alpha} \nabla u_{j}-P_{h} u_{j}\right\|_{L_{2}\left(\partial R_{j}\right)}^{2}\right)^{1 / 2}\left(\sum_{j}\left\|w-m_{j}\right\|_{L_{2}\left(\partial R_{j}\right)}^{2}\right)^{1 / 2} \\
& \leq C h^{k-3 / 2}\|u\|_{k}\left(\sum_{j}\left\|w-m_{j}\right\|_{L_{2}\left(R_{j}\right)}\left\|w-m_{j}\right\|_{H_{1}\left(R_{j}\right)}\right)^{1 / 2} \\
& \leq C h^{k-3 / 2}\|u\|_{k} h^{1 / 2}\|w\|_{h} \\
& =C h^{k-1}\|u\|_{k}\|w\|_{h}, \quad k=2,3,4 .
\end{aligned}
$$

Thus, we have

$$
\sup _{w \in \mathscr{N} \mathscr{C}_{0}^{h}}\left\{\frac{\left|a_{h}(u, w)-\langle f, w\rangle\right|}{\|w\|_{h}}\right\} \leq C h^{k-1}\|u\|_{H^{k}(\Omega)}, \quad k=2,3,4 .
$$

By collecting the above results, we get the following energy-norm error estimate.

Theorem 3. Let $u \in H^{k+1}(\Omega) \cap H_{0}^{1}(\Omega)$ and $u_{h} \in \mathscr{N}_{C_{0}^{h}}$ be the solution of (8) and (9), respectively. Then we have

$$
\left\|u-u_{h}\right\|_{h} \leq c h^{k}\|u\|_{H^{k+1}(\Omega)}, \quad k=1,2,3 .
$$

By the standard Aubin-Nitsche duality argument, the $L_{2}(\Omega)$-error estimate can be easily obtained, but the corresponding proof is omitted. We state the result in the following theorem.

Theorem 4. Let $u \in H^{k+1}(\Omega) \cap H_{0}^{1}(\Omega)$ and $u_{h} \in \mathscr{N} \mathscr{C}_{0}^{h}$ be the solution of (8) and (9), respectively. Then we have

$$
\left\|u-u_{h}\right\|_{0} \leq C h^{k+1}\|u\|_{H^{k+1}(\Omega)}, \quad k=1,2,3 .
$$

Instead of the Dirichlet problem, if the following Neumann problem

$$
\begin{aligned}
-\nabla \cdot(\boldsymbol{\alpha} \nabla u)+\beta u=f, & \Omega, \\
\nu \cdot(\boldsymbol{\alpha} \nabla u)+\gamma u=g, & \Gamma,
\end{aligned}
$$

is considered, the weak problem (8) is then replaced by finding $u \in H^{1}(\Omega)$ such that

$$
a^{n}(u, v)=(f, v)+\langle g, v\rangle, \quad v \in H^{1}(\Omega),
$$

where $a^{n}$ is the bilinear form defined by $a^{n}(u, v)=(\boldsymbol{\alpha} \nabla u, \nabla v)+(\beta u, v)+\langle\gamma u, v\rangle$ for all $u, v \in H^{1}(\Omega)$, and $\langle\cdot, \cdot\rangle$ is the paring between $H^{-1 / 2}(\Gamma)$ and $H^{1 / 2}(\Gamma)$. Thus, the nonconforming method for Problem (15) states as follows: find $u_{h} \in \mathscr{N} \mathscr{C}^{h}$ such that

$$
a_{h}^{n}\left(u_{h}, v_{h}\right)=\left(f, v_{h}\right)+\left\langle g, v_{h}\right\rangle, \quad v_{h} \in \mathscr{N} \mathscr{C}^{h} .
$$

Then all the arguments given above for Dirichlet case hold analogously Hence one can have the following result.

Theorem 5. Let $u \in H^{k+1}(\Omega)$ and $u_{h} \in \mathscr{N} \mathscr{C}^{h}$ satisfy (16) and (17), respectively. Then we have the energy-norm and $L^{2}$-norm error estimates:

$$
\begin{aligned}
& \left\|u-u_{h}\right\|_{h} \leq C h^{k}\|u\|_{H^{k+1}(\Omega)}, \\
& \left\|u-u_{h}\right\|_{0} \leq C h^{k+1}\|u\|_{H^{k+1}(\Omega)}, \quad k=1,2,3 .
\end{aligned}
$$




\section{Numerical examples}

In this section we illustrate two numerical examples. First, consider the following Dirichlet problem:

$$
\begin{array}{r}
-\Delta u=f, \quad \Omega, \\
u=0, \quad \Gamma,
\end{array}
$$

where $\Omega=(0,1)^{2}$. The source term $f$ is calculated from the the exact solution

$$
u(x, y)=\sin (2 \pi x) \sin (2 \pi y)\left(x^{3}-y^{4}+x^{2} y^{3}\right) .
$$

Table 1 shows the numerical results, where the error reduction ratios in $L_{2}(\Omega)$ and broken energy norm are optimal.

\begin{tabular}{|r|c|c|c|c|c|}
\hline \hline $\mathrm{h}$ & DOFs & $\left\|u-u_{h}\right\|_{0}$ & ratio & $\left\|u-u_{h}\right\|_{h}$ & ratio \\
\hline $1 / 2$ & 9 & 0.148 & - & 1.759 & - \\
$1 / 4$ & 57 & $1.200 \mathrm{E}-002$ & 3.62 & 0.300 & 2.55 \\
$1 / 8$ & 273 & $4.690 \mathrm{E}-004$ & 4.68 & $3.051 \mathrm{E}-002$ & 3.30 \\
$1 / 16$ & 1185 & $2.292 \mathrm{E}-005$ & 4.35 & $3.355 \mathrm{E}-003$ & 3.19 \\
$1 / 32$ & 4929 & $1.279 \mathrm{E}-006$ & 4.16 & $3.940 \mathrm{E}-004$ & 3.09 \\
$1 / 64$ & 20097 & $7.590 \mathrm{E}-008$ & 4.08 & $4.78 \mathrm{E}-005$ & 3.04 \\
$1 / 128$ & 81153 & $4.629 \mathrm{E}-009$ & 4.04 & $5.881 \mathrm{E}-006$ & 3.02 \\
\hline \hline
\end{tabular}

Table 1: The Dirichlet problem: The apparent $L^{2}$ and broken energy norm errors and their reduction ratios on the quadrilateral meshes.

Next, turn to the following Neumann problem:

$$
\begin{aligned}
-\Delta u+u & =f, \quad \Omega, \\
\frac{\partial u}{\partial n} & =g, \quad \Gamma,
\end{aligned}
$$

with the same domain $\Omega=(0,1)^{2}$. The source terms $f$ and $g$ are are generated from the exact solution

$$
u(x, y)=\cos (2 \pi x) \cos (2 \pi y)\left(x^{3}-y^{4}+x^{2} y^{3}\right) .
$$

Again, Table 2 shows the numerical results, where the error reduction ratios in $L_{2}(\Omega)$ and broken energy norm are optimal.

\begin{tabular}{|r|c|c|c|c|c|}
\hline \hline $\mathrm{h}$ & DOFs & $\left\|u-u_{h}\right\|_{0}$ & ratio & $\left\|u-u_{h}\right\|_{h}$ & ratio \\
\hline $1 / 2$ & 32 & $3.850 \mathrm{E}-002$ & - & 0.698 & - \\
$1 / 4$ & 104 & $5.217 \mathrm{E}-003$ & 2.88 & 0.172 & 2.02 \\
$1 / 8$ & 368 & $3.325 \mathrm{E}-004$ & 3.97 & $2.348 \mathrm{E}-002$ & 2.88 \\
$1 / 16$ & 1376 & $1.917 \mathrm{E}-005$ & 4.12 & $2.907 \mathrm{E}-003$ & 3.01 \\
$1 / 32$ & 5312 & $1.162 \mathrm{E}-006$ & 4.04 & $3.616 \mathrm{E}-004$ & 3.01 \\
$1 / 64$ & 20864 & $7.201 \mathrm{E}-008$ & 4.01 & $4.513 \mathrm{E}-005$ & 3.00 \\
$1 / 128$ & 82688 & $4.491 \mathrm{E}-009$ & 4.00 & $5.639 \mathrm{E}-006$ & 3.00 \\
\hline \hline
\end{tabular}

Table 2: The Neumann problem: The apparent $L^{2}$ - and broken energy norm errors and their reduction ratios on the quadrilateral meshes. 


\section{References}

[1] D. N. Arnold and R. Winther. Nonconforming mixed elements for elasticity. Dedicated to Jim Douglas, Jr. on the occasion of his 75th birthday. Math. Models Methods Appl. Sci., 13(3):295-307, 2003.

[2] G. Awanou. A rotated nonconforming rectangular mixed element for elasticity. Calcolo, 46:49-60, 2009. 10.1007/s10092-009-0159-6.

[3] I. Babuška and M. Suri. Locking effect in the finite element approximation of elasticity problem. Numer. Math., 62:439-463, 1992.

[4] I. Babuška and M. Suri. On locking and robustness in the finie element method. SIAM J. Numer. Anal., 29:1261-1293, 1992.

[5] S. C. Brenner and L. Y. Sung. Linear finite element methods for planar elasticity. Math. Comp., 59:321-338, 1992.

[6] F. Brezzi and M. Fortin. Mixed and Hybrid Finite Element Methods, volume 15 of Springer Series in Computational Mathematics. Springer-Verlag, New York, 1991.

[7] Z. Cai, J. Douglas, Jr., J. E. Santos, D. Sheen, and X. Ye. Nonconforming quadrilateral finite elements: A correction. Calcolo, 37(4):253-254, 2000.

[8] Z. Cai, J. Douglas, Jr., and X. Ye. A stable nonconforming quadrilateral finite element method for the stationary Stokes and Navier-Stokes equations. Calcolo, 36:215-232, 1999.

[9] P. G. Ciarlet. The Finite Element Method for Elliptic Equations. North-Holland, Amsterdam, 1978.

[10] M. Crouzeix and P.-A. Raviart. Conforming and nonconforming finite element methods for solving the stationary Stokes equations. R.A.I.R.O.- Math. Model. Anal. Numer., R-3:33-75, 1973.

[11] R. S. Falk. Nonconforming finite element methods for the equations of linear elasticity. Math. Comp., 57:529-550, 1991.

[12] M. Farhloul and M. Fortin. A mixed nonconforming finite element for the elasticity and Stokes problems. Math. Models Methods Appl. Sci., 9(8):1179-1199, 1999.

[13] M. Fortin. A three-dimensional quadratic nonconforming element. Numer. Math., 46:269-279, 1985.

[14] M. Fortin and M. Soulie. A non-conforming piecewise quadratic finite element on the triangle. Int. J. Numer. Meth. Engrg., 19(4):505-520, 1983.

[15] V. Girault and P.-A. Raviart. Finite Element Methods for Navier-Stokes Equations, Theory and Algorithms. Springer-Verlag, Berlin, 1986.

[16] J. Hu and Z.C. Shi. Lower order rectangular nonconforming mixed finite elements for plane elasticity. SIAM J. Numer. Anal, 46(1):88-102, 2007.

[17] B.H. Irons and A. Razzaque. Experience with the patch test for convergence of finite elements. In A.K.Aziz, editor, The mathematical foundations of the finite element method with applications to partial differential equations, pages 557-587, New York, 1977. Academic Press.

[18] I. Kim, Z. Luo, Z. Meng, H. Nam, C. Park, and D. Sheen. A piecewise $p_{2}$-nonconforming quadrilateral finite element. M2AN: Mathematical Modelling and Numerical Analysis. in press. 2013.

[19] P. Klouček, B. Li, and M. Luskin. Analysis of a class of nonconforming finite elements for crystalline microstructures. Math. Comp., 65(215):1111-1135, 1996. 
[20] C.-O. Lee, J. Lee, and D. Sheen. A locking-free nonconforming finite element method for planar elasticity. Adv. Comput. Math., 19(1-3):277-291, 2003.

[21] H. Lee and D. Sheen. A new quadratic nonconforming finite element on rectangles. Numer. Methods Partial Differential Equations, 22(4):954-970, 2006.

[22] B. Li and M. Luskin. Nonconforming finite element approximation of crystalline microstructure. Math. Comp., 67(223):917-946, 1998.

[23] P. Ming and Z.-C. Shi. Nonconforming rotated $Q_{1}$ element for Reissner-Mindlin plate. Math. Models Methods Appl. Sci., 11(8):1311-1342, 2001.

[24] C. Park and D. Sheen. $p_{1}$-nonconforming quadrilateral finite element methods for second-order elliptic problems. SIAM Journal on Numerical Analysis, 41(2):624-640, 2004.

[25] R. Rannacher and S. Turek. Simple nonconforming quadrilateral Stokes element. Numer. Methods Partial Differential Equations, 8:97-111, 1992.

[26] G. Strang and G. J. Fix. An Analysis of the Finite Element Method. Prentice-Hall, Englewood Cliffs, 1973.

[27] S.-Y. Yi. Nonconforming mixed finite element methods for linear elasticity using rectangular elements in two and three dimensions. Calcolo, 42:115-133, 2005. 10.1007/s10092-005-0101-5.

[28] Z. Zhang. Analysis of some quadrilateral nonconforming elements for incompressible elasticity. SIAM J. Numer. Anal., 34(2):640-663, 1997. 